\title{
Breakout Prediction Based on BP Neural Network in Continuous Casting Process
}

\author{
ZHANG Ben-guo $^{1, \mathrm{a}}$, ZHANG Xinjiang ${ }^{1, \mathrm{~b}}$ and FAN Lifeng ${ }^{2, \mathrm{c}}$ \\ ${ }^{1}$ Yancheng institute technology, Yancheng 264005, China \\ ${ }^{2}$ Inner Mongolia University, Hohhot, 010070, China \\ azhangbenguo@163.com, bzhangxinjiang1983@163.com, '251155328@qq.com
}

\begin{abstract}
An improved BP neural network model was presented by modifying the learning algorithm of the traditional BP neural network, based on the Levenberg-Marquardt algorithm, and was applied to the breakout prediction system in the continuous casting process. The results showed that the accuracy rate of the model for the temperature pattern of sticking breakout was $96.43 \%$, and the quote rate was $100 \%$, that verified the feasibility of the model.
\end{abstract}

Keywords: continuous casting; breakout prediction; BP neural network

\section{Introduction}

Continuous Casting is the process whereby molten steel is solidified into a semi finished slab, or some such elementary form, for subsequent rolling in the finishing mills $^{[1]}$. Breakouts are of major concern in the continuous steel-casting process, because they can lead to severe damage to equipment, significant process downtime, and potential safety consequences. The loss caused by a typical breakout accident is close to 200,000 dollars ${ }^{[2]}$. Among many forms of breakouts, the sticking-type breakout has the highest incidence and the greatest harm, thus it is very critical to prevent the sticking type breakout occurrence for reducing incidence of breakout accident.

In this paper, based on the indepth study of a variety of breakout prediction systems, a breakout prediction system has been constructed based on a BP (Back Propagation) neural network model which was optimized with LM (Levenberg Marquardt) algorithm. And the system was trained and tested with the data collected at the continuous casting production site.

\section{Principle of Sticking-type Breakout Prediction}

The prediction of the sticking type breakout is to make the right judgments of the temperature patterns which may cause leakage. The principal of the means is to identify the abnormal temperature waves from normal ones measured by thermocouples embedded in the inner walls of the casting mould. Actually, it is a dynamic wave pattern recognition process, as shown in Fig. 1. Under normal continuous casting conditions, the temperature reading of thermocouples decreases gradually with the growth of the solidified shell' thickness, and the temperature reading measured by the thermocouples in the first row is higher than the temperature reading measured by the second row thermocouples in the same vertical plane, as shown in Fig. 1(a). The temperatures measured by thermocouples imbedded in the mould at each measuring point are relatively stable and can only fluctuate narrowly, as shown in Fig. 1(b). At the continuous casting progresses, the sticker occurs in the vicinity of the meniscus and remains during the negative strip time. A tear in the shell can then occur at the sticking point during the positive strip time when the forces acting on the shell exceed the shear strength of the shell. When the sticker reaches the upper thermocouple mounted close to the meniscus, the temperature reading rises, as shown in Fig. 1(c). At the point of the sticker, the strand sticks to the side of the mould more strongly than elsewhere under the action of the stresses in the strand shell, with the result that the speed of the shell is also reduced at sticking point. The temperatures measured by the upper thermocouples decrease with the gradual healing of tear, and the temperatures measured by the lower thermocouples rise with the crack propagation, as shown in Fig. 1(d). Once the sticker goes past the upper thermocouples, the temperature readings of them begin decreasing, such that it will be smaller than the lower thermocouple reading at some point. Then the breakout alarm is sent out, as shown in Fig. 1(e). As the sticker goes past the lower thermocouple its temperature reading begins decreasing too, as shown in Fig. 1(f). 


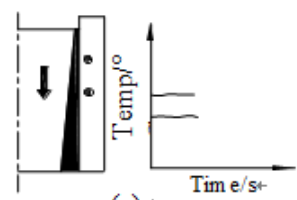

(a)

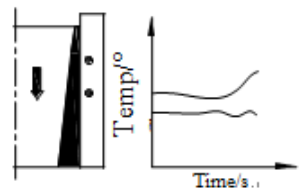

(c)

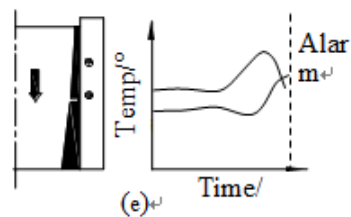

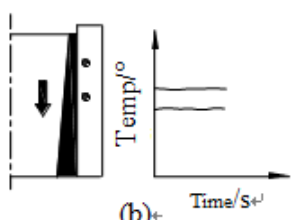

(b)+ Time/s

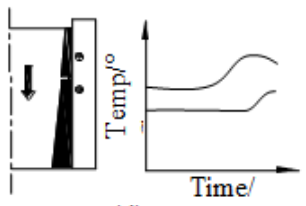

(d)

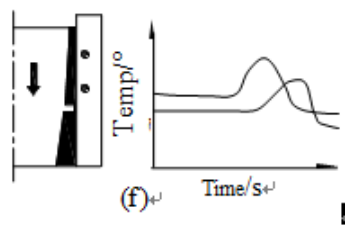

Fig. 1 The principle of sticking type breakout prediction in continuous casting process

\section{Breakout Prediction Based on BP Neural Network of LM Algorithm}

Currently, BP neural network is the most widely applied neural network, and 80\% $90 \%$ artificial neural network models in practice are BP neural networks or its changes in various forms. Therefore, BP neural network is the core part of feed-forward neural networks, and is the most essential artificial neural network $^{[3]}$. That anyone of continuous functions in a closed interval can be approximated by a BP network with one hidden layer has been proved by Robert Hecht-Nielson in 1989, thus any n-dimension to $\mathrm{m}$ dimensional mapping can be completed with a threelayer BP network ${ }^{[4]}$. In this paper, the structure of the BP neural network with the LM algorithm as the learning algorithm is the same as the standard BP network (see Fig.2).

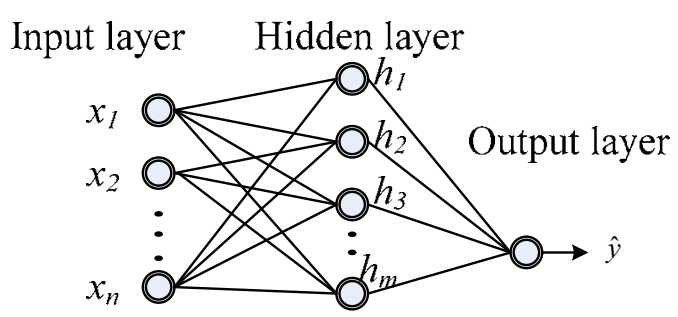

Fig.2 Three layers BP net

\section{BP Neural Network of LM Algorithm}

The algorithm of the standard BP is based on the gradient descent algorithm. In order for the network to learn, the value of each weight and threshold has to be incrementally adjusted proportionally to the contribution of each unit compared with the total error.
The learning process is composed by forward and backward information disseminations: input sample data forward transmission and error information feedback. The mean squared errors of the expectations and the output of neural network tend to be minimized by adjusting the weights and thresholds ${ }^{[5]}$. It is actually to make a minimum mean square error of an approximate gradient descent algorithm. There are some shortcomings in the convergence process, e.g. slow rate of convergence, existing local minimum.

At present, there are two main methods for increasing the convergence rate of the BP network: (1) using heuristic information technology, e.g. adding momentum term to the learning algorithm; (2) using numerical optimization methods, e.g. conjugate gradient method, LM algorithm, etc. Although the BP algorithm using heuristic technology is simple and intuitive and to some extent can improve the convergence speed, its accuracy achievable is limited. Among the numerical optimization methods, LM algorithm has the fastest convergence rate and the smallest training error. Therefore, LM algorithm is chosen as an optimization algorithm for the BP neural network in the breakout prediction system. The iterative formula for the BP neural network is shown as follows:

$$
w(n+1)=w(n)-\left[J^{T} J+u I\right]^{-1} \cdot J^{T} e(n)
$$

Where $\mathrm{J}$ is the Jacobian matrix, as follows:

$$
J=\left|\frac{\partial e(n)}{\partial w_{1}} \frac{\partial e(n)}{\partial w_{2}} \cdots \frac{\partial e(n)}{\partial w_{i}}\right|
$$

The calculation steps are as follows:

1) Give the initialize weights and the value of training error allowed: $\varepsilon, \beta, \mu$, set $n=0, u=u_{0}$.

2) Calculate the output of the network $y(n)$ and the error $e(n)$.

3) Calculate the Jacobian matrix J.

4) Calculate the rectification $\Delta w(n)$ of the weights, and change the weights.

5) If $e(n)<\varepsilon$, turn to step (7), otherwise, set $w(n+1)$ as the weight and calculate the error $e(n+1)$.

6) If $e(n+1)<e(n)$, set $n=n+1, \mu=\mu / \beta$, and turn to step (2); otherwise, don't change the weights this time, set $w(n+1)=w(n), u=u \beta$, turn to step (4).

7) Stop.

\section{Data Preprocessing}

In order to eliminate the effects of the order of magnitude on the output of the neural network and highlight the date with Breakout features, the data are normalized as follows: 


$$
X^{*}(i)= \begin{cases}\frac{X(i)}{\sqrt{\sum_{i=1}^{15} X^{2}(i)}} & \left(X_{\max }-X_{\min }\right) \leq \lambda \\ \frac{X(i)-X_{\min }}{X_{\text {max }}-X_{\min }} & \left(X_{\text {max }}-X_{\min }\right)>\lambda\end{cases}
$$

Where, $X=\left\{X_{1}, X_{2}, \ldots X_{n}\right\}$ is the raw data, $n$ is the dimension of the input column vector, and $\lambda$ is the stability threshold.The temperature sequence is considered to be stable, if the temperature change is not greater than $\lambda$. Otherwise, the temperature sequence is considered to be volatile. Usually, the change of the temperature is greater than $35^{\circ} \mathrm{C}$, when the breakout occurs. this paper takes $\lambda=25^{\circ} \mathrm{C}$ through the analysis of historical data collected in a steel plant.

Using the general data normalization method would undermine this stability, and it is not conducive to the identification of the network model. So, the equation (3) is used for the data normalization in this paper, and that problem is well resolved.

\section{The Determining of the Network Parameters}

1) The nodes in the input layer and hidden layer: It is found that the time for the temperature fluctuation measured by a single thermocouple is about 30 s during the breakout process. Considering the size and the sensitivity of the neural network, the number of the nodes in the input layer and the output layer is set to 15 and 1 . The output of the neural network indicated the likelihood of the breakout.

2) The determination of the optimal number of hidden layer neurons: According to Kolmogorov's theorem, the approximate relationship between the number of the input layer neurons and the number of the hidden layer neurons in three-layer neural network is:

$$
K \leq 2 m+1
$$

Where $\mathrm{K}$ is the number of the hidden layer neurons and $m$ is the number of the input layer neurons. So the number of the neurons in the hidden layer is less than or equal to 31 according to Eq.(4). In this paper, through repeated experiments, results analysis and comparison, the hidden layer nodes is determined as 30 .

\section{Training of the Neural Network Model}

1) First of all, 100 groups of typical temperature trace containing 30 groups of normal and 70 groups with breakout pattern have been chosen from the historical data of the temperature trace collected from a continuous casting plant as the training samples for the neural network model.

2) The maximum training times is set as 1000 , the maximum allowable error is set as $10^{-6}$, and the learning rate is set as 0.1 .

3) The Neural Network Training Result is shown as Fig. 3:

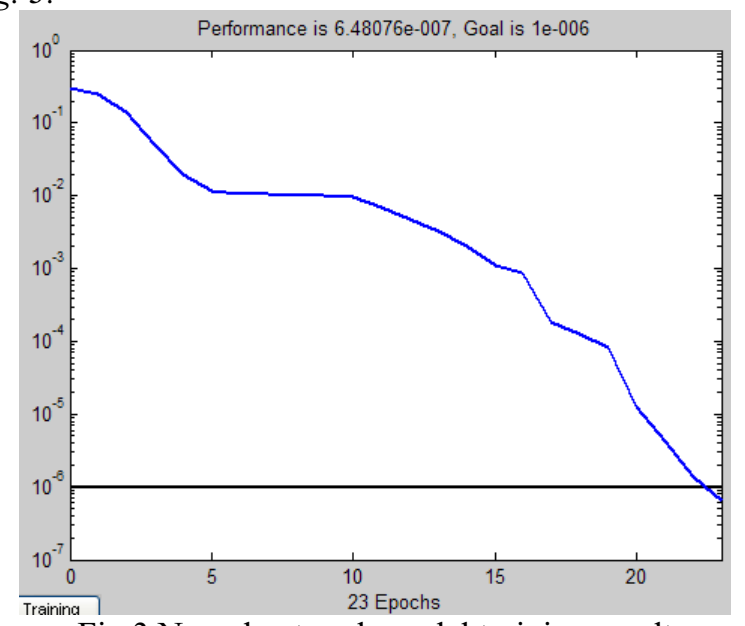

Fig 3 Neural network model training result

\section{Testing of the Neural Network Model}

The testing samples for the neural network model were 56 groups of typical temperature trace collected from the continuous casting plant, containing 27 groups of sticking type breakout samples, 9 groups of false alarm samples, and 20 groups of normal samples. In the period of testing, all the 27 true breakout alarms, and 1 case of undesirable alarm were sent out by the model. So the accuracy rate of the model for the temperature pattern of sticking breakout was $96.43 \%$, and the quote rate was $100 \%$.

\section{Conclusions}

In this paper, the improved BP neural network model based on the LM algorithm was presented by modifying the learning algorithm of the traditional BP neural network. It has stronger real-time efficiency and faster convergence speed, compared with the traditional BP neural network model. The model was applied to the breakout prediction system in the continuous casting process; the identification capabilities and the forecast accuracy were greatly improved. The result of the test was that the accuracy rate of the model for the temperature pattern of sticking breakout was $96.43 \%$, and the quote rate was $100 \%$. The feasibility of the model for the breakout prediction system was well verified.

\section{Acknowledgment}

The authors would like to acknowledge the support of Basic Research Program of Jiangsu province (No. BK20150429). 


\section{References}

1. Zhang B, Zhang R, Wang G, et al. Breakout prediction for continuous casting using genetic algorithm-based back propagation neural network model. Int. J. Modeling, Identification and Control, 2012,16(3):199-205.

2. Irani R, Nasimi R. Evolving neural network using real coded genetic algorithm for permeability estimation of the reservoir[J]. Expert Systems with Applications, 2011,38(8): 9862-9866.

3. Li Zhang, Jianhua Luo, Suying Yang. Forecasting box office revenue of movies with BP neural network J. Expert Systems with Applications, vol. 36, 2009, pp. 6580-6587.

4. Q. Ju, Z. Yu, Z. Hao, et al. Hydrologic simulations with artificial neural networks, in: Third International Conference on Natural Computation, IEEE Computer Society Publications, vol. 2, 2007, pp. 22-27.

5. S.H. Hsiang and Y.W. Lin. Application of fuzzy theory to predict deformation behaviors of magnesium alloy sheets under hot extrusion, Journal of Materials Processing Technology 201 (2008), pp. 138-144. 